ANNALES

POLONICI MATHEMATICI

$84.2(2004)$

\title{
Numerical approximations of parabolic differential functional equations with the initial boundary conditions of the Neumann type
}

\author{
by Roman Ciarski (Gdańsk)
}

\begin{abstract}
The aim of this paper is to present a numerical approximation for quasilinear parabolic differential functional equations with initial boundary conditions of the Neumann type. The convergence result is proved for a difference scheme with the property that the difference operators approximating mixed derivatives depend on the local properties of the coefficients of the differential equation. A numerical example is given.
\end{abstract}

1. Introduction. We will denote by $C(U, V)$ the class of all continuous functions $w: U \rightarrow V$ with $U$ and $V$ being any metric spaces. Let $M_{n \times n}$ be the set of $n \times n$ matrices with real elements. For $x=\left(x_{1}, \ldots, x_{n}\right) \in \mathbb{R}^{n}$ and $X \in M_{n \times n}, X=\left[X_{k j}\right]_{j, k=1}^{n}$, we put

$$
\|x\|=\left|x_{1}\right|+\cdots+\left|x_{n}\right|, \quad\|X\|=\max \left\{\sum_{j=1}^{n}\left|x_{k j}\right|: 1 \leq k \leq n\right\} .
$$

Writing a vectorial inequality we mean that the same inequality holds for the corresponding components. Let $a>0, \mathbb{R}_{+}=[0,+\infty)$, and $b=\left(b_{1}, \ldots, b_{n}\right)$ $\in \mathbb{R}^{n}$ be given, where $b_{k}>0$ for $1 \leq k \leq n$. Define

$$
\begin{aligned}
E & =[0, a] \times(-b, b), & E_{0} & =\{0\} \times[-b, b], \\
\partial_{0} E & =([0, a] \times[-b, b]) \backslash E, & E^{*} & =E_{0} \cup E \cup \partial_{0} E .
\end{aligned}
$$

Assume that

$$
\varrho=\left[\varrho_{k j}\right]_{j, k=1}^{n}: E \times C\left(E^{*}, \mathbb{R}\right) \rightarrow M_{n \times n}, \quad f: E \times C\left(E^{*}, \mathbb{R}\right) \times \mathbb{R}^{n} \rightarrow \mathbb{R}
$$

are given functions of the variables $(t, x, w)$ and $(t, x, w, p)$ respectively.

We consider a quasilinear differential functional equation with Neumann initial boundary conditions

2000 Mathematics Subject Classification: Primary 65M12; Secondary 35R10.

Key words and phrases: parabolic differential functional equation, Neumann boundary condition. 


$$
\left\{\begin{array}{l}
\partial_{t} z(t, x)=\sum_{k, j=1}^{n} \varrho_{k j}(t, x, z) \partial_{x_{k} x_{j}} z(t, x)+f\left(t, x, z, \partial_{x} z(t, x)\right), \\
z(t, x)=\varphi_{0}(t, x) \quad \text { for }(t, x) \in E_{0}, \\
\partial_{x_{j}} z(t, x)=\varphi_{j}(t, x) \quad \text { for }(t, x) \in \partial_{0} E \text { and } x_{j}=b_{j} \text { or } x_{j}=-b_{j},
\end{array}\right.
$$

where $\varphi_{0}: E_{0} \rightarrow \mathbb{R}$ and $\varphi_{j}: \partial_{0} E \rightarrow \mathbb{R}, 1 \leq j \leq n$, are given and $\partial_{x} z=$ $\left(\partial_{x_{1}} z, \ldots, \partial_{x_{n}} z\right)$.

The difference methods for nonlinear parabolic equations with Neumann boundary conditions were initiated in the papers by Malec [4]-[6] and Węglowski [8]. In [1], some general difference operators were introduced and their stability was investigated. The results of [4]-[6] and [8] do not apply to quasilinear equations. The difference scheme applied in this paper has the property that the difference operators approximating the mixed derivatives depend on the local properties of the function $\varrho$. We give sufficient conditions for convergence of the difference method for problem (1). The convergence is proved by consistency and stability arguments. We are interested in the numerical approximation of a classical solution of the above problem.

The norm of any $z \in C\left(E^{*}, \mathbb{R}\right)$ is defined by

$$
\|z\|_{E^{*}}=\max \left\{|z(t, x)|:(t, x) \in E^{*}\right\} .
$$

We will need the norm

$$
\|z\|_{t}=\max \left\{|z(\theta, x)|: 0 \leq \theta \leq t \text { and }(\theta, x) \in E^{*}\right\} .
$$

For $t \in[0, a]$ we write $H_{t}=[0, t] \times[-b, b]$. We assume that problem (1) is of Volterra type, that is, if $t \in[0, a]$ and $z, \bar{z} \in C\left(E^{*}, \mathbb{R}\right)$ and $z(\theta)=\bar{z}(\theta)$ for $\theta \in H_{t}$ then $f(t, x, z, q)=f(t, x, \bar{z}, q)$ and $\varrho_{k j}(t, x, z)=\varrho_{k j}(t, x, \bar{z})$ for $x \in[-b, b], q \in \mathbb{R}^{n}$ and $j, k=1, \ldots, n$.

2. Difference functional equations. Let $\mathbb{N}$ and $\mathbb{Z}$ be the sets of natural numbers and integers respectively. For $x, \bar{x} \in \mathbb{R}^{n}, x=\left(x_{1}, \ldots, x_{n}\right)$, $\bar{x}=\left(\bar{x}_{1}, \ldots, \bar{x}_{n}\right)$, we put $x * \bar{x}=\left(x_{1} \bar{x}_{1}, \ldots, x_{n} \bar{x}_{n}\right)$. We define a mesh on the set $E^{*}$ in the following way. Suppose that $h=\left(h_{0}, h^{\prime}\right) \in \mathbb{R}_{+}^{1+n}$, where $h^{\prime}=\left(h_{1}, \ldots, h_{n}\right)$ are the steps of the mesh. Denote by $\Delta$ the set of all $h=\left(h_{0}, h^{\prime}\right)$ such that there exist $N_{b}=\left(N_{b 1}, \ldots, N_{b n}\right) \in \mathbb{Z}^{n}$ with $N_{b} * h^{\prime}=b$. For $h \in \Delta$ we write $\|h\|=h_{0}+h_{1}+\cdots+h_{n}$ and $\left\|h^{\prime}\right\|=h_{1}+\cdots+h_{n}$. It is required that $\Delta \neq \emptyset$ and that there exists a sequence $\left\{h^{(j)}\right\}$ in $\Delta$ such that $\lim _{j \rightarrow \infty}\left\|h^{(j)}\right\|=0$.

Nodal points are defined by:

$$
t^{(i)}=i h_{0}, \quad x^{(m)}=m * h=\left(m_{1} h_{1}, \ldots, m_{n} h_{n}\right)=\left(x_{1}^{\left(m_{1}\right)}, \ldots, x_{n}^{\left(m_{n}\right)}\right),
$$

where $(i, m) \in \mathbb{Z}^{1+n}$. Obviously there exists $N_{a} \in \mathbb{N}$ such that $N_{a} h_{0} \leq a$ 
$<\left(N_{a}+1\right) h_{0}$. Let

$$
R_{h}^{1+n}=\left\{\left(t^{(i)}, x^{(m)}\right):(i, m) \in \mathbb{Z}^{1+n}\right\}
$$

and

$$
\begin{aligned}
E_{h} & =\bar{E} \cap R_{h}^{1+n}, & \partial_{0} E_{h} & =\partial_{0} E \cap R_{h}^{1+n}, \\
E_{0 \cdot h} & =E_{0} \cap R_{h}^{1+n}, & E_{h}^{*} & =E_{h} \cup E_{0 \cdot h} \cup \partial_{0} E_{h} .
\end{aligned}
$$

For $z: E_{h}^{*} \rightarrow \mathbb{R}$ we write

$$
z^{(i, m)}=z\left(t^{(i)}, x^{(m)}\right) .
$$

The norm of any $z: E_{h}^{*} \rightarrow \mathbb{R}$ is defined by

$$
\|z\|_{h}=\max \left\{\left|z^{(i, m)}\right|:\left(t^{(i)}, x^{(m)}\right) \in E_{h}^{*}\right\} .
$$

For any $t^{(i)}$ we will need the norm

$$
\|z\|_{h \cdot i}=\max \left\{\left|z^{(r, m)}\right|: 0 \leq r \leq i \text { and }\left(t^{(r)}, x^{(m)}\right) \in E_{h}^{*}\right\} .
$$

Let

$$
E_{h}^{\prime}=\left\{\left(t^{(i)}, x^{(m)}\right) \in E_{h}: 0 \leq i \leq N_{a}-1\right\}
$$

and denote by $\mathfrak{F}\left(E_{h}^{*}, \mathbb{R}\right)$ the set of all functions $w: E_{h}^{*} \rightarrow \mathbb{R}$. Suppose that $\varrho_{h}=\left[\varrho_{h \cdot k j}\right]_{j, k=1}^{n}: E_{h}^{\prime} \times \mathfrak{F}\left(E_{h}^{*}, \mathbb{R}\right) \rightarrow M_{n \times n}, \quad f_{h}: E_{h}^{\prime} \times \mathfrak{F}\left(E_{h}^{*}, \mathbb{R}\right) \times \mathbb{R}^{n} \rightarrow \mathbb{R}$,
$\varphi_{0 \cdot h}: E_{0 \cdot h} \rightarrow \mathbb{R}, \quad \varphi_{j \cdot h}: \partial_{0} E_{h} \rightarrow \mathbb{R}, \quad j=1, \ldots, n$,

are given functions. We will approximate solutions of problem (1) by means of solutions of a difference equation with initial boundary condition of Neumann type. To do that, for every $\left(t^{(i)}, x^{(m)}\right) \in \partial_{0} E_{h}$ we define

$$
\begin{gathered}
\mathcal{A}^{(m)}=\left\{\alpha=\left(\alpha_{1}, \ldots, \alpha_{n}\right): \alpha_{j} \in\{0,1\} \text { if } x_{j}^{\left(m_{j}\right)}=b_{j},\right. \\
\alpha_{j} \in\{0,-1\} \text { if } x_{j}^{\left(m_{j}\right)}=-b_{j}, \\
\alpha_{j}=0 \text { if }-b_{j}<x_{j}^{\left(m_{j}\right)}<b_{j}, \\
\text { and }\|\alpha\|=1 \text { or }\|\alpha\|=2,1 \leq j \leq n\},
\end{gathered}
$$

where $\|\alpha\|=\left|\alpha_{1}\right|+\cdots+\left|\alpha_{n}\right|$, and

$$
\begin{aligned}
\partial E_{h}^{+1} & =\left\{\left(t^{(i)}, x^{(m+\alpha)}\right): 0 \leq i \leq N_{a},\left(t^{(i)}, x^{(m)}\right) \in \partial_{0} E_{h} \text { and } \alpha \in \mathcal{A}^{(m)}\right\}, \\
E_{h}^{+1} & =\partial E_{h}^{+1} \cup E_{h} .
\end{aligned}
$$

Now we consider the difference problem

(2) $\delta_{0} z^{(i, m)}=\sum_{k, j=1}^{n} \varrho_{h \cdot k j}\left(t^{(i)}, x^{(m)}, z\right) \delta_{k j}^{(2)} z^{(i, m)}+f_{h}\left(t^{(i)}, x^{(m)}, z, \delta z^{(i, m)}\right)$ 
with Neumann boundary conditions

$$
\begin{gathered}
z^{(i, m)}=\varphi_{0 \cdot h}^{(i, m)} \quad \text { on } E_{0 \cdot h}, \\
z_{h}^{(i, m+\alpha)}-z_{h}^{(i, m-\alpha)}=2 \sum_{j=1}^{n} \alpha_{j} h_{j} \varphi_{j \cdot h}^{(i, m)} \quad \text { on } \partial_{0} E_{h} \text { for } \alpha \in \mathcal{A}^{(m)} .
\end{gathered}
$$

Let us notice that $\left(t^{(i)}, x^{(m+\alpha)}\right) \in \partial E_{h}^{+1}$ and $\left(t^{(i)}, x^{(m-\alpha)}\right) \in E_{h}$.

Let $e_{j} \in \mathbb{R}^{n}$ be the standard unit vectors. The difference operators $\delta_{0}$, $\delta=\left(\delta_{1}, \ldots, \delta_{n}\right), \delta_{\alpha}$ and $\delta^{(2)}=\left[\delta_{k j}\right]_{j, k=1}^{n}$ are defined in the following way:

$$
\begin{array}{rlrl}
\delta_{0} z^{(i, m)} & =\frac{1}{h_{0}}\left[z^{(i+1, m)}-z^{(i, m)}\right], \\
\delta_{j} z^{(i, m)} & =\frac{1}{2 h_{j}}\left[z^{\left(i, m+e_{j}\right)}-z^{\left(i, m-e_{j}\right)}\right], & & 1 \leq j \leq n, \\
\delta_{k k}^{(2)} z^{(i, m)} & =\delta_{k}^{+} \delta_{k}^{-} z^{(i, m)}, \\
\delta_{k j}^{(2)} z^{(i, m)} & =\frac{1}{2}\left[\delta_{k}^{+} \delta_{j}^{+} z^{(i, m)}+\delta_{k}^{-} \delta_{j}^{-} z^{(i, m)}\right] & & \text { if } \varrho_{h \cdot k j}\left(t^{(i)}, x^{(m)}, z\right) \geq 0, \\
\delta_{k j}^{(2)} z^{(i, m)} & =\frac{1}{2}\left[\delta_{k}^{+} \delta_{j}^{-} z^{(i, m)}+\delta_{k}^{-} \delta_{j}^{+} z^{(i, m)}\right] & & \text { if } \varrho_{h \cdot k j}\left(t^{(i)}, x^{(m)}, z\right)<0,
\end{array}
$$

where

$$
\delta_{k}^{+} z^{(i, m)}=\frac{1}{h_{k}}\left[z^{\left(i, m+e_{k}\right)}-z^{(i, m)}\right], \quad \delta_{k}^{-} z^{(i, m)}=\frac{1}{h_{k}}\left[z^{(i, m)}-z^{\left(i, m-e_{k}\right)}\right] .
$$

There exists exactly one solution $u_{h}: E^{*} \rightarrow \mathbb{R}$ of problem (2)-(4). Let the operator $F_{h}$ be defined by

$$
F_{h}[z]^{(i, m)}=\sum_{k, j=1}^{n} \varrho_{h \cdot k j}\left(t^{(i)}, x^{(m)}, z\right) \delta_{k j} z^{(i, m)}+f_{h}\left(t^{(i)}, x^{(m)}, z, \delta z^{(i, m)}\right) .
$$

Our purpose is to examine the relation between the solution $u_{h}$ of $(2)-(4)$ and a function $v_{h}: E_{h}^{+1} \rightarrow \mathbb{R}$ satisfying the condition

$$
\begin{aligned}
\left|\delta_{0} v_{h}^{(i, m)}-F_{h}\left[v_{h}\right]^{(i, m)}\right| & \leq \gamma(h) & & \text { on } E_{h}^{\prime}, \\
\left|v_{h}^{(i, m)}-\varphi_{0 \cdot h}^{(i, m)}\right| & \leq \gamma_{0}(h) & & \text { on } E_{0 \cdot h}, \\
\left|v_{h}^{(i, m+\alpha)}-v_{h}^{(i, m-\alpha)}-2 \sum_{j=1}^{n} \alpha_{j} h_{j} \varphi_{j \cdot h}^{(i, m)}\right| & \leq C_{\varphi}\left\|h^{\prime}\right\|^{3} & & \text { on } \partial_{0} E_{h},
\end{aligned}
$$

where

$$
\gamma, \gamma_{0}: \Delta \rightarrow \mathbb{R}_{+}, \quad \lim _{h \rightarrow 0} \gamma_{0}(h)=0, \quad \lim _{h \rightarrow 0} \gamma(h)=0, \quad C_{\varphi} \in \mathbb{R}_{+}, \quad \alpha \in \mathcal{A}^{(m)} .
$$

The function $v_{h}$ satisfying the above relations is considered to be an approximate solution of problem (2)-(4). 
Assumption $\mathrm{H}\left[\varrho_{h}, f_{h}\right]$. The functions $\varrho_{h}: E_{h}^{\prime} \times \mathfrak{F}\left(E_{h}^{*}, \mathbb{R}\right) \rightarrow M_{n \times n}$ and $f_{h}: E_{h}^{\prime} \times \mathfrak{F}\left(E_{h}^{*}, \mathbb{R}\right) \times \mathbb{R}^{n} \rightarrow \mathbb{R}$ satisfy the following conditions:

(i) there exists $L \in \mathbb{R}_{+}$such that

$$
\begin{array}{r}
\left\|\varrho_{h}\left(t^{(i)}, x^{(m)}, w\right)-\varrho_{h}\left(t^{(i)}, x^{(m)}, \bar{w}\right)\right\| \leq L\|w-\bar{w}\|_{h \cdot i}, \\
\left|f_{h}\left(t^{(i)}, x^{(m)}, w, p\right)-f_{h}\left(t^{(i)}, x^{(m)}, \bar{w}, p\right)\right| \leq L\|w-\bar{w}\|_{h \cdot i},
\end{array}
$$

(ii) the derivative $\partial_{p} f_{h}=\left(\partial_{p_{1}} f_{h}, \ldots, \partial_{p_{n}} f_{h}\right)$ exists on $E_{h}^{\prime} \times \mathfrak{F}\left(E_{h}^{*}, \mathbb{R}\right) \times \mathbb{R}^{n}$ and $\partial_{p} f_{h}(t, x, w, \cdot) \in C\left(\mathbb{R}^{n}, \mathbb{R}^{n}\right)$.

Theorem 1. Suppose that Assumption $\mathrm{H}\left[\varrho_{h}, f_{h}\right]$ holds and

(i) $h \in \Delta$ and

$$
\begin{gathered}
1-2 h_{0} \sum_{k=1}^{n} \frac{1}{h_{k}^{2}} \varrho_{h \cdot k k}(Q)+h_{0} \sum_{\substack{k, j=1 \\
j \neq k}}^{n} \frac{1}{h_{k} h_{j}}\left|\varrho_{h \cdot k j}(Q)\right| \geq 0, \\
\frac{1}{h_{k}} \varrho_{h \cdot k k}(Q)-\sum_{\substack{j=1 \\
j \neq k}}^{n} \frac{1}{h_{j}}\left|\varrho_{h \cdot k j}(Q)\right|-\frac{1}{2}\left|\partial_{p_{k}} f_{h}(P)\right| \geq 0, \quad 1 \leq k \leq n,
\end{gathered}
$$

where $Q=(t, x, w) \in E_{h}^{\prime} \times \mathfrak{F}\left(E_{h}^{*}, \mathbb{R}\right)$ and $P=(t, x, w, p) \in E_{h}^{\prime} \times \mathfrak{F}\left(E_{h}^{*}, \mathbb{R}\right) \times \mathbb{R}^{n}$,

(ii) $u_{h}: E_{h}^{+1} \rightarrow \mathbb{R}$ is the solution of problem (2)-(4),

(iii) $v_{h}: E_{h}^{+1} \rightarrow \mathbb{R}$ satisfies relations (11)-(13),

(iv) there exists $c_{0} \in \mathbb{R}_{+}$such that

$$
\left|\delta_{k j}^{(2)} v_{h}^{(i, m)}\right| \leq c_{0} \quad \text { on } E_{h} \text { for } 1 \leq k, j \leq n,
$$

(v) there exists $\widetilde{C} \in \mathbb{R}_{+}$such that $\left\|h^{\prime}\right\|^{2} \leq \widetilde{C} h_{0}$.

Under these assumptions we have

if $L>0$, and

$$
\left|u_{h}^{(i, m)}-v_{h}^{(i, m)}\right| \leq \gamma_{0}(h) e^{\widetilde{L} a}+\beta(h) \frac{e^{\widetilde{L} a}-1}{\widetilde{L}} \quad \text { on } E_{h}
$$

$$
\left|u_{h}^{(i, m)}-v_{h}^{(i, m)}\right| \leq \gamma_{0}(h)+a \gamma(h) \quad \text { on } E_{h}
$$

if $L=0$, where $\widetilde{L}=L\left(1+n c_{0}\right)$ and

$$
\beta: \Delta \rightarrow \mathbb{R}_{+}, \quad \lim _{h \rightarrow 0} \beta(h)=0 .
$$

Proof. Let $\Gamma: E_{h}^{\prime} \rightarrow \mathbb{R}, \Gamma_{0 \cdot h}: E_{0 \cdot h} \rightarrow \mathbb{R}$ and $\Gamma_{\partial \cdot h}: \partial_{0} E_{h} \rightarrow \mathbb{R}$ be defined by

$$
\begin{aligned}
\delta_{0} v_{h}^{(i, m)} & =F_{h}\left[v_{h}\right]^{(i, m)}+\Gamma_{h}^{(i, m)} & & \text { on } E_{h}^{\prime}, \\
v_{h}^{(i, m)} & =\varphi_{0 \cdot h}^{(i, m)}+\Gamma_{0 \cdot h}^{(i, m)} & & \text { on } E_{0 \cdot h}, \\
v_{h}^{(i, m+\alpha)}-v_{h}^{(i, m-\alpha)} & =2 \sum_{j=1}^{n} \alpha_{j} h_{j} \varphi_{j \cdot h}^{(i, m)}+\Gamma_{\partial \cdot h}^{(i, m)} & & \text { on } \partial_{0} E_{h} \text { for } \alpha \in \mathcal{A}^{(m)} .
\end{aligned}
$$


Then

$$
\begin{array}{ll}
\left|\Gamma_{h}^{(i, m)}\right| \leq \gamma(h) & \text { on } E_{h}^{\prime} \text { with } \lim _{h \rightarrow 0} \gamma(h)=0, \\
\left|\Gamma_{0 \cdot h}^{(i, m)}\right| \leq \gamma_{0}(h) & \text { on } E_{0 \cdot h} \text { with } \lim _{h \rightarrow 0} \gamma_{0}(h)=0, \\
\left|\Gamma_{\partial \cdot h}^{(i, m)}\right| \leq C_{\varphi}\left\|h^{\prime}\right\|^{3} & \text { on } \partial_{0} E_{h} .
\end{array}
$$

The function $\varepsilon_{h}=u_{h}-v_{h}$ satisfies the difference functional equation

$$
\begin{aligned}
& \delta_{0} \varepsilon_{h}^{(i, m)}=\sum_{k, j=1}^{n} \varrho_{h \cdot k j}\left(t^{(i)}, x^{(m)}, u_{h}\right) \delta_{k j}^{(2)} \varepsilon_{h}^{(i, m)} \\
& \quad+f_{h}\left(t^{(i)}, x^{(m)}, v_{h}, \delta u_{h}^{(i, m)}\right)-f_{h}\left(t^{(i)}, x^{(m)}, v_{h}, \delta v_{h}^{(i, m)}\right)+\Lambda_{h}^{(i, m)}
\end{aligned}
$$

where

$$
\begin{aligned}
\Lambda_{h}^{(i, m)} & =\sum_{k, j=1}^{n}\left[\varrho_{h \cdot k j}\left(t^{(i)}, x^{(m)}, u_{h}\right)-\varrho_{h \cdot k j}\left(t^{(i)}, x^{(m)}, v_{h}\right)\right] \delta_{k j}^{(2)} v_{h}^{(i, m)} \\
& +f_{h}\left(t^{(i)}, x^{(m)}, u_{h}, \delta u_{h}^{(i, m)}\right)-f_{h}\left(t^{(i)}, x^{(m)}, v_{h}, \delta u_{h}^{(i, m)}\right)-\Gamma_{h}^{(i, m)}
\end{aligned}
$$

on $E_{h}^{\prime}$ and

$$
\varepsilon_{h}^{(i, m+\alpha)}=\varepsilon_{h}^{(i, m-\alpha)}+\Gamma_{\partial \cdot h}^{(i, m)}
$$

on $\partial_{0} E_{h}^{\prime}$. Let us deal with $\varepsilon_{h}$ on $E_{h}^{\prime}$ first. Write

$$
\begin{aligned}
& I_{+}^{(i, m)}=\left\{(k, j): 1 \leq k, j \leq n, k \neq j, \varrho_{h \cdot k j}\left(t^{(i)}, x^{(m)}, u_{h}\right) \geq 0\right\}, \\
& I_{-}^{(i, m)}=\left\{(k, j): 1 \leq k, j \leq n, k \neq j, \varrho_{h \cdot k j}\left(t^{(i)}, x^{(m)}, u_{h}\right)<0\right\} .
\end{aligned}
$$

From (5) and the mean value theorem, we can rewrite (18) as

$$
\begin{aligned}
\varepsilon_{h}^{(i+1, m)}=\varepsilon_{h}^{(i, m)} & +h_{0} \sum_{k=1}^{n} \varrho_{h \cdot k k}\left(t^{(i)}, x^{(m)}, u_{h}\right) \delta_{k k}^{(2)} \varepsilon_{h}^{(i, m)} \\
& +h_{0} \sum_{(k, j) \in I_{+}^{(i, m)}} \varrho_{h \cdot k j}\left(t^{(i)}, x^{(m)}, u_{h}\right) \delta_{k j}^{(2)} \varepsilon_{h}^{(i, m)} \\
& +h_{0} \sum_{(k, j) \in I_{-}^{(i, m)}} \varrho_{h \cdot k j}\left(t^{(i)}, x^{(m)}, u_{h}\right) \delta_{k j}^{(2)} \varepsilon_{h}^{(i, m)} \\
& +h_{0} \sum_{k=1}^{n} \partial_{p_{k}} f_{h}(P) \frac{\varepsilon_{h}^{\left(i, m+e_{k}\right)}-\varepsilon_{h}^{\left(i, m-e_{k}\right)}}{2 h_{k}}+h_{0} \Lambda_{h}^{(i, m)}
\end{aligned}
$$

By (7)-(9) and regrouping terms, the function $\varepsilon_{h}$ satisfies on $E_{h}^{\prime}$ the recursive 
equation

$$
\begin{aligned}
\varepsilon_{h}^{(i+1, m)}= & \varepsilon_{h}^{(i, m)} \boldsymbol{A}^{(i, m)}+h_{0} \sum_{k=1}^{n} \varepsilon_{h}^{\left(i, m+e_{k}\right)} \boldsymbol{B}_{k}^{(i, m)}+h_{0} \sum_{k=1}^{n} \varepsilon_{h}^{\left(i, m-e_{k}\right)} \boldsymbol{C}_{k}^{(i, m)}(21) \\
& +h_{0} \sum_{(k, j) \in I_{+}^{(i, m)}}\left[\varepsilon_{h}^{\left(i, m+e_{k}+e_{j}\right)}+\varepsilon_{h}^{\left(i, m-e_{k}-e_{j}\right)}\right] \boldsymbol{D}_{k, j}^{(i, m)} \\
& +h_{0} \sum_{(k, j) \in I_{-}^{(i, m)}}\left[\varepsilon_{h}^{\left(i, m+e_{k}-e_{j}\right)}+\varepsilon_{h}^{\left(i, m-e_{k}+e_{j}\right)}\right] \boldsymbol{D}_{k, j}^{(i, m)}+h_{0} \Lambda_{h}^{(i, m)},
\end{aligned}
$$

where

$$
\begin{aligned}
\boldsymbol{A}^{(i, m)}= & 1-2 h_{0} \sum_{k=1}^{n} \frac{1}{h_{k}^{2}} \varrho_{h \cdot k k}\left(t^{(i)}, x^{(m)}, u_{h}\right) \\
& +h_{0} \sum_{\substack{k, j=1 \\
j \neq k}}^{n} \frac{1}{h_{k} h_{j}}\left|\varrho_{h \cdot k j}\left(t^{(i)}, x^{(m)}, u_{h}\right)\right|, \\
\boldsymbol{B}_{k}^{(i, m)}= & \frac{1}{h_{k}^{2}} \varrho_{h \cdot k k}\left(t^{(i)}, x^{(m)}, u_{h}\right) \\
& -\sum_{\substack{j=1 \\
j \neq k}}^{n} \frac{1}{h_{k} h_{j}}\left|\varrho_{h \cdot k j}\left(t^{(i)}, x^{(m)}, u_{h}\right)\right|+\frac{1}{2 h_{k}} \partial_{p_{k}} f_{h}(P), \\
\boldsymbol{C}_{k}^{(i, m)}= & \frac{1}{h_{k}^{2}} \varrho_{h \cdot k k}\left(t^{(i)}, x^{(m)}, u_{h}\right) \\
& -\sum_{\substack{j=1 \\
j \neq k}}^{n} \frac{1}{h_{k} h_{j}}\left|\varrho_{h \cdot k j}\left(t^{(i)}, x^{(m)}, u_{h}\right)\right|-\frac{1}{2 h_{k}} \partial_{p_{k}} f_{h}(P), \\
& \frac{1}{2 h_{k} h_{j}}\left|\varrho_{h \cdot k j}\left(t^{(i)}, x^{(m)}, u_{h}\right)\right| .
\end{aligned}
$$

Let $\omega_{h}$ and $\widetilde{\omega}_{h}$ be given by

$$
\begin{gathered}
\omega_{h}^{(i)}=\max \left\{\left|\varepsilon_{h}^{(r, m)}\right|:\left(t^{(r)}, x^{(m)}\right) \in E_{h}^{*} \cap\left(\left[-\tau_{0}, t^{(i)}\right] \times \mathbb{R}^{n}\right)\right\}, \\
0 \leq i \leq N_{a}, \\
\widetilde{\omega}_{h}^{(i)}=\max \left\{\left|\varepsilon_{h}^{(r, m)}\right|:\left(t^{(r)}, x^{(m)}\right) \in E_{h}^{+1} \cap\left(\left[-\tau_{0}, t^{(i)}\right] \times \mathbb{R}^{n}\right)\right\}, \\
0 \leq i \leq N_{a} .
\end{gathered}
$$

With this notation $\Lambda_{h}$ (see (19)) can be estimated as follows:

$$
\left|\Lambda_{h}^{(i, m)}\right| \leq \widetilde{\omega}_{h}^{(i)} L\left(1+n c_{0}\right)+\gamma(h) \quad \text { on } E_{h}^{\prime}
$$


We conclude from (12), (20), (21) and (24) that the functions $\omega_{h}$ and $\widetilde{\omega}_{h}$ satisfy the recursive inequalities

$$
\begin{aligned}
\omega_{h}^{(i+1)} & \leq \widetilde{\omega}_{h}^{(i)}\left(1+\widetilde{L} h_{0}\right)+h_{0} \gamma(h), \\
\widetilde{\omega}_{h}^{(i)} & \leq \omega_{h}^{(i)}+\sqrt{h_{0}} h_{0} C_{\varphi} \widetilde{C}
\end{aligned}
$$

for $0 \leq i \leq N_{a}-1$ and

$$
\omega_{h}^{(0)} \leq \gamma_{0}(h)
$$

Consider the difference equations

$$
\begin{aligned}
\eta_{h}^{(i+1)} & =\widetilde{\eta}_{h}^{(i)}\left(1+\widetilde{L} h_{0}\right)+h_{0} \gamma(h), \\
\widetilde{\eta}_{h}^{(i)} & =\eta_{h}^{(i)}+\sqrt{h_{0}} h_{0} C_{\varphi} \widetilde{C},
\end{aligned}
$$

for $0 \leq i \leq N_{a}-1$ with the initial condition

$$
\eta^{(0)}=\gamma_{0}(h)
$$

and its solutions

$$
\begin{aligned}
& \eta_{h}^{(0)}=\gamma_{0}(h) \\
& \widetilde{\eta}_{h}^{(0)}=\gamma_{0}(h)+\sqrt{h_{0}} h_{0} C_{\varphi} \widetilde{C} \\
& \eta_{h}^{(i)}=\gamma_{0}(h)\left(1+\widetilde{L} h_{0}\right)^{i}+h_{0}\left[\left(1+\widetilde{L} h_{0}\right) \sqrt{h_{0}} C_{\varphi} \widetilde{C}+\gamma(h)\right] \sum_{j=0}^{i-1}\left(1+\widetilde{L} h_{0}\right)^{j},
\end{aligned}
$$

for $1 \leq i \leq N_{a}$. Thus

$$
\eta_{h}^{(i)} \leq \gamma_{0}(h) e^{\widetilde{L} a}+\left[\left(1+\widetilde{L} h_{0}\right) \sqrt{h_{0}} C_{\varphi} \widetilde{C}+\gamma(h)\right] \frac{e^{\widetilde{L} a}-1}{\widetilde{L}} .
$$

It follows from (25)-(27) that

$$
\omega_{h}^{(i)} \leq \eta_{h}^{(i)} \quad \text { for } 0 \leq i \leq N_{a} .
$$

This gives (16), (17) and Theorem 1 is proved.

3. Difference method for the mixed problem. We will need an interpolating operator $T_{h}: \mathfrak{F}\left(E_{h}^{*}, \mathbb{R}\right) \rightarrow C(E, \mathbb{R})$. Let

$$
S_{+}=\left\{\xi=\left(\xi_{1}, \ldots, \xi_{n}\right): \xi_{j}=\{0,1\} \text { for } 0 \leq j \leq n\right\}
$$

Let $z \in \mathfrak{F}\left(E_{h}^{*}, \mathbb{R}\right)$. For every $(t, x) \in E$ there is $\left(t^{(i)}, x^{(m)}\right) \in E_{h}$ such that $\left(t^{(i+1)}, x^{(m+1)}\right) \in E_{h}^{\prime}$, where $m+1=\left(m_{1}+1, \ldots, m_{n}+1\right)$ and $t^{(i)} \leq t \leq t^{(i+1)}$, $x^{(m)} \leq x \leq x^{(m+1)}$. Set 


$$
\begin{aligned}
\left(T_{h} z\right)(t, x)= & \frac{t-t^{(i)}}{h_{0}} \sum_{\xi \in S_{+}} z^{(i+1, m+\xi)}\left(\frac{x-x^{(m)}}{h^{\prime}}\right)^{\xi}\left(1-\frac{x-x^{(m)}}{h^{\prime}}\right)^{1-\xi} \\
& +\left(1-\frac{t-t^{(i)}}{h_{0}}\right) \sum_{\xi \in S_{+}} z^{(i, m+\xi)}\left(\frac{x-x^{(m)}}{h^{\prime}}\right)^{\xi}\left(1-\frac{x-x^{(m)}}{h^{\prime}}\right)^{1-\xi}
\end{aligned}
$$

where

$$
\begin{gathered}
\left(\frac{x-x^{(m)}}{h^{\prime}}\right)^{\xi}=\prod_{j=1}^{n}\left(\frac{x_{j}-x_{j}^{\left(m_{j}\right)}}{h_{j}}\right)^{\xi_{j}}, \\
\left(1-\frac{x-x^{(m)}}{h^{\prime}}\right)^{1-\xi}=\prod_{j=1}^{n}\left(1-\frac{x_{j}-x_{j}^{\left(m_{j}\right)}}{h_{j}}\right)^{1-\xi_{j}} .
\end{gathered}
$$

In the above formulas we adopt the convention that $0^{0}=1$. For $h_{0} N_{a}<t \leq a$ we put

$$
\left(T_{h} z\right)(t, x)=\left(T_{h} z\right)\left(h_{0} N_{a}, x\right) .
$$

Lemma 2. Suppose that

(i) $z(t, \cdot):[-b, b] \rightarrow \mathbb{R}$ is of class $C^{2}$ for $t \in[0, a]$ and $z_{h}=\left.z\right|_{E_{h}^{*}}$,

(ii) $\widetilde{d}_{2} \in \mathbb{R}_{+}$is such that on $E^{*}$,

$$
\left|\partial_{x_{j} x_{k}} z(t, x)\right| \leq \widetilde{d}_{2} \quad \text { for } j, k=1, \ldots, n,
$$

(iii) there exists $\widetilde{c} \in \mathbb{R}_{+}$such that $h_{0}<\widetilde{c}\left\|h^{\prime}\right\|^{2}$,

(iv) there is $L \in \mathbb{R}_{+}$such that

$$
|z(t, x)-z(\bar{t}, x)| \leq L|t-\bar{t}| .
$$

Then

$$
\left\|T_{h} z_{h}-z\right\|_{E^{*}} \leq C_{0}\left\|h^{\prime}\right\|^{2},
$$

where $C_{0}=\widetilde{d}_{2}+2 L \widetilde{c}$ and $\left\|h^{\prime}\right\|=h_{1}+\cdots+h_{n}$.

Proof. Let $(t, x) \in E$ and $(i, m)$ be such that $t^{(i)} \leq t \leq t^{(i+1)}$ and $x^{(m)} \leq x \leq x^{(m+1)}$. Then

$$
\begin{array}{r}
\left(T_{h} z\right)(t, x)-z(t, x) \\
=\sum_{\xi \in S_{+}} z^{(i, m+\xi)}\left(\frac{x-x^{(m)}}{h^{\prime}}\right)^{\xi}\left(1-\frac{x-x^{(m)}}{h^{\prime}}\right)^{1-\xi}-z\left(t^{(i)}, x\right) \\
+\left(\frac{t-t^{(i)}}{h_{0}}\right) \sum_{\xi \in S_{+}}\left[z^{(i+1, m+\xi)}-z^{(i, m+\xi)}\right]\left(\frac{x-x^{(m)}}{h^{\prime}}\right)^{\xi}\left(1-\frac{x-x^{(m)}}{h^{\prime}}\right)^{1-\xi} \\
+z\left(t^{(i)}, x\right)-z(t, x) .
\end{array}
$$


It is easy to prove by induction that

$$
\sum_{\xi \in S_{+}}\left(\frac{x-x^{(m)}}{h^{\prime}}\right)^{\xi}\left(1-\frac{x-x^{(m)}}{h^{\prime}}\right)^{1-\xi}=1
$$

and

$$
\sum_{\xi \in S_{+}}\left(\frac{x-x^{(m)}}{h^{\prime}}\right)^{\xi}\left(1-\frac{x-x^{(m)}}{h^{\prime}}\right)^{1-\xi} \xi_{k} h_{k}=x_{k}-x_{k}^{\left(m_{k}\right)} .
$$

Thus, it is easily seen that

$$
\begin{array}{r}
\left|\left(\frac{t-t^{(i)}}{h_{0}}\right) \sum_{\xi \in S_{+}}\left[z^{(i+1, m+\xi)}-z^{(i, m+\xi)}\right]\left(\frac{x-x^{(m)}}{h^{\prime}}\right)^{\xi}\left(1-\frac{x-x^{(m)}}{h^{\prime}}\right)^{1-\xi}\right| \\
\leq L \widetilde{c}\left\|h^{\prime}\right\|^{2}
\end{array}
$$

and

$$
\left|z\left(t^{(i)}, x\right)-z(t, x)\right| \leq L \widetilde{c}\left\|h^{\prime}\right\|^{2} .
$$

Finally, by the Taylor formula, (28), (32) and (33) we obtain

$$
\left|\sum_{\xi \in S_{+}} z^{(i, m+\xi)}\left(\frac{x-x^{(m)}}{h^{\prime}}\right)^{\xi}\left(1-\frac{x-x^{(m)}}{h^{\prime}}\right)^{1-\xi}-z\left(t^{(i)}, x\right)\right| \leq \widetilde{d}_{2}\left\|h^{\prime}\right\|^{2} .
$$

From (31) and (34)-(36) we get

$$
\left|\left(T_{h} z\right)(t, x)-z(t, x)\right| \leq \widetilde{d_{2}}\left\|h^{\prime}\right\|^{2}+2 L \widetilde{c}\left\|h^{\prime}\right\|^{2},
$$

which implies assertion (30).

Assumption $\mathrm{H}[\varrho, f]$. Suppose that

(i) $\varrho: E^{\prime} \times C(E, \mathbb{R}) \rightarrow M_{n \times n}$ and $f: E \times C(E, \mathbb{R}) \times \mathbb{R}^{n} \rightarrow \mathbb{R}$ are continuous,

(ii) there exists $L \in \mathbb{R}_{+}$such that

$$
\begin{aligned}
\|\varrho(t, x, w)-\varrho(t, x, \bar{w})\| & \leq L\|w-\bar{w}\|_{t}, \\
|f(t, x, w, p)-f(t, x, \bar{w}, p)| & \leq L\|w-\bar{w}\|_{t},
\end{aligned}
$$

(iii) $\partial_{p} f=\left(\partial_{p_{1}} f, \ldots, \partial_{p_{n}} f\right)$ exists on $E \times C(E, \mathbb{R}) \times \mathbb{R}^{n}$ and $\partial_{p} f(t, x, w, \cdot)$ $\in C\left(\mathbb{R}^{n}, \mathbb{R}^{n}\right)$.

Now we will approximate the solution of the functional differential problem (1) by the solution of the difference problem

$$
\begin{aligned}
& \delta_{0} z^{(i, m)} \\
& \quad=\sum_{k, j=1}^{n} \varrho_{h \cdot k j}\left(t^{(i)}, x^{(m)}, T_{h} z\right) \delta_{k j}^{(2)} z^{(i, m)}+f_{h}\left(t^{(i)}, x^{(m)}, T_{h} z, \delta z^{(i, m)}\right)
\end{aligned}
$$


with the initial Neumann boundary conditions

$$
\begin{gathered}
z^{(i, m)}=\varphi_{0}^{(i, m)} \quad \text { on } E_{0 \cdot h}, \\
z_{h}^{(i, m+\alpha)}-z_{h}^{(i, m-\alpha)}=2 \sum_{j=1}^{n} \alpha_{j} h_{j} \varphi_{j \cdot h}^{(i, m)} \\
\quad \text { for }\left(t^{(i)}, x^{(m)}\right) \in \partial_{0} E_{h} \text { and } \alpha \in \mathcal{A}^{(m)} .
\end{gathered}
$$

Let $\Omega$ be an open set such that $E^{*} \subset \Omega$.

Theorem 3. Suppose that assumption $\mathrm{H}[\varrho, f]$ holds and

(i) $h \in \Delta$ and

$$
\begin{array}{r}
1-2 h_{0} \sum_{k=1}^{n} \frac{1}{h_{k}^{2}} \varrho_{k k}(t, x, w)+h_{0} \sum_{\substack{k, j=1 \\
j \neq k}}^{n} \frac{1}{h_{k} h_{j}}\left|\varrho_{k j}(t, x, w)\right| \geq 0, \\
\frac{1}{h_{k}} \varrho_{k k}(t, x, w)-\sum_{\substack{j=1 \\
j \neq k}}^{n} \frac{1}{h_{j}}\left|\varrho_{k j}(t, x, w)\right|-\frac{1}{2}\left|\partial_{p_{k}} f(t, x, w, p)\right| \geq 0, \\
1 \leq k \leq n
\end{array}
$$

(ii) there exists $c_{\star} \in \mathbb{R}_{+}$such that $h_{k} \leq c_{\star} h_{j}$ for $1 \leq k, j \leq n$,

(iii) there exist $\widetilde{C}, \widetilde{c} \in \mathbb{R}_{+}$such that $\widetilde{C}^{-1}\left\|h^{\prime}\right\|^{2} \leq h_{0} \leq \widetilde{c}\left\|h^{\prime}\right\|^{2}$,

(iv) $u_{h}: E_{h}^{+1} \rightarrow \mathbb{R}$ is a solution of (37)-(39),

(v) $v: \Omega \rightarrow \mathbb{R}$ is a solution of (1) on $E^{*}$ and $v_{h}=\left.v\right|_{E_{h}^{*}}, \varphi_{j \cdot h}=\left.\varphi_{j}\right|_{\partial_{0} E_{h}}$, $1 \leq j \leq n$,

(vi) there exists $\gamma_{0}: \Delta \rightarrow \mathbb{R}_{+}$such that

$$
\left|\varphi_{0}^{(i, m)}-\varphi_{0 \cdot h}^{(i, m)}\right| \leq \gamma_{0}(h) \quad \text { on } E_{0 \cdot h}, \quad \lim _{h \rightarrow 0} \gamma_{0}(h)=0,
$$

(vii) $v(\cdot, x)$ is of class $C^{1}$ and $v(t, \cdot)$ is of class $C^{3}$.

Then there is $\eta: \Delta \rightarrow \mathbb{R}_{+}$such that on $E_{h}$,

$$
\left|u_{h}^{(i, m)}-v_{h}^{(i, m)}\right| \leq \eta(h), \quad \lim _{h \rightarrow 0} \eta(h)=0 .
$$

Proof. We apply Theorem 1. Write

$$
\begin{aligned}
\psi_{h}^{(i, m)}= & \delta_{0} v_{h}^{(i, m)}-\sum_{k, j=1}^{n} \varrho_{k j}\left(t^{(i)}, x^{(m)}, T_{h} v_{h}\right) \delta_{k j}^{(2)} v_{h}^{(i, m)} \\
& -f\left(t^{(i)}, x^{(m)}, T_{h} v_{h}, \delta v_{h}^{(i, m)}\right), \\
\xi_{h}^{(i, m)}= & v_{h}^{(i, m+\alpha)}-v_{h}^{(i, m-\alpha)} v-2 \sum_{j=1}^{n} \alpha_{j} h_{j} \varphi_{j \cdot h}^{(i, m)} .
\end{aligned}
$$


We see at once that on $E_{h}^{\prime}$,

$$
\begin{aligned}
\psi_{h}^{(i, m)}= & \delta_{0} v_{h}^{(i, m)}-\partial_{t} v\left(t^{(i)}, x^{(m)}\right) \\
& +\sum_{k, j=1}^{n}\left[\varrho_{k j}\left(t^{(i)}, x^{(m)}, v\right)-\varrho_{k j}\left(t^{(i)}, x^{(m)}, T_{h} v_{h}\right)\right] \delta_{k j}^{(2)} v_{h}^{(i, m)} \\
& +\sum_{k, j=1}^{n} \varrho_{k j}\left(t^{(i)}, x^{(m)}, v\right)\left[\partial_{x_{k} x_{j}}^{(2)} v^{(i, m)}-\delta_{k j}^{(2)} v_{h}^{(i, m)}\right] \\
& +f\left(t^{(i)}, x^{(m)}, v, \partial_{x} v\left(t^{(i)}, x^{(m)}\right)\right)-f\left(t^{(i)}, x^{(m)}, T_{h} v_{h}, \partial_{x} v\left(t^{(i)}, x^{(m)}\right)\right) \\
& +f\left(t^{(i)}, x^{(m)}, T_{h} v_{h}, \partial_{x} v\left(t^{(i)}, x^{(m)}\right)\right)-f\left(t^{(i)}, x^{(m)}, T_{h} v_{h}, \delta v_{h}^{(i, m)}\right) .
\end{aligned}
$$

It is easily seen that there is $\gamma: \Delta \rightarrow \mathbb{R}_{+}$such that

$$
\left|\psi_{h}^{(i, m)}\right| \leq \gamma\left(h \quad \text { on } E_{h}^{\prime}, \quad \lim _{h \rightarrow 0} \gamma(h)=0 .\right.
$$

Now on $\partial E_{h}$ we have

$$
\xi_{h}^{(i, m)}=v_{h}^{(i, m+\alpha)}-v_{h}^{(i, m-\alpha)}-2 \sum_{j=1}^{n} \alpha_{j} h_{j} \varphi_{j \cdot h}^{(i, m)} .
$$

By the Taylor formula we get

$$
\begin{aligned}
v^{(i, m+\alpha)}- & v^{(i, m-\alpha)}-2 \sum_{j=1}^{n} \alpha_{j} h_{j} \varphi_{j \cdot h}^{(i, m)} \\
= & v^{(i, m)}+\sum_{j=1}^{n} \alpha_{j} h_{j} \partial_{x_{j}} v^{(i, m)}+\frac{1}{2} \sum_{k, j=1}^{n} \alpha_{j} \alpha_{k} h_{j} h_{k} \partial_{x_{j} x_{k}}^{(2)} v^{(i, m)} \\
& +\frac{1}{6} \sum_{j, k, l=1}^{n} \alpha_{j} \alpha_{k} \alpha_{l} h_{j} h_{k} h_{l} \partial_{x_{j} x_{k} x_{l}}^{(3)} v(P) \\
& -\left[v^{(i, m)}-\sum_{j=1}^{n} \alpha_{j} h_{j} \partial_{x_{j}} v^{(i, m)}+\frac{1}{2} \sum_{k, j=1}^{n} \alpha_{j} \alpha_{k} h_{j} h_{k} \partial_{x_{j} x_{k}}^{(2)} v^{(i, m)}\right. \\
& \left.-\frac{1}{6} \sum_{j, k, l=1}^{n} \alpha_{j} \alpha_{k} \alpha_{l} h_{j} h_{k} h_{l} \partial_{x_{j} x_{k} x_{l}}^{(3)} v(Q)\right]-2 \sum_{j=1}^{n} \alpha_{j} h_{j} \varphi_{j \cdot h}^{(i, m)} \\
= & \frac{1}{6} \sum_{j, k, l=1}^{n} \alpha_{j} \alpha_{k} \alpha_{l} h_{j} h_{k} h_{l}\left[\partial_{x_{j} x_{k} x_{l}}^{(3)} v(P)-\partial_{x_{j} x_{k} x_{l}}^{(3)} v(Q)\right]
\end{aligned}
$$

and finally

$$
\left|\xi_{h}^{(i, m)}\right| \leq \widetilde{d}_{3}\left|\sum_{j, k, l=1}^{n} \alpha_{j} \alpha_{k} \alpha_{l} h_{j} h_{k} h_{l}\right| \leq \widetilde{d_{3}}\left\|h^{\prime}\right\|^{3}
$$


where $\widetilde{d}_{3} \in \mathbb{R}_{+}$. Thus, all the assumptions of Theorem 1 are satisfied and assertion (41) follows from (16), (17).

Now we give an error estimate for the method (37)-(39).

TheOrem 4. Suppose that assumption $\mathrm{H}[\varrho, f]$ holds and

(i) $h \in \Delta$ and

$$
\begin{gathered}
1-2 h_{0} \sum_{k=1}^{n} \frac{1}{h_{k}^{2}} \varrho_{k k}(t, x, w)+h_{0} \sum_{\substack{k, j=1 \\
j \neq k}}^{n} \frac{1}{h_{k} h_{j}}\left|\varrho_{k j}(t, x, w)\right| \geq 0, \\
\frac{1}{h_{k}} \varrho_{k k}(t, x, w)-\sum_{\substack{j=1 \\
j \neq k}}^{n} \frac{1}{h_{j}}\left|\varrho_{k j}(t, x, w)\right|-\frac{1}{2}\left|\partial_{p_{k}} f(t, x, w, p)\right| \geq 0, \\
\quad 1 \leq k \leq n,
\end{gathered}
$$

(ii) there exists $c_{\star} \in \mathbb{R}_{+}$such that $h_{k} \leq c_{\star} h_{j}$ for $1 \leq k, j \leq n$,

(iii) there exist $\widetilde{C}, \widetilde{c} \in \mathbb{R}_{+}$such that $\widetilde{C}^{-1}\left\|h^{\prime}\right\|^{2} \leq h_{0} \leq \widetilde{c}\left\|h^{\prime}\right\|^{2}$,

(iv) $u_{h}: E_{h}^{+1} \rightarrow \mathbb{R}$ is a solution of (37)-(39),

(v) $v: \Omega \rightarrow \mathbb{R}$ is a solution of (1) on $E^{*}$ and $v_{h}=\left.v\right|_{E_{h}^{*}}, \varphi_{j \cdot h}=\left.\varphi_{j}\right|_{\partial_{0} E_{h}}$, $1 \leq j \leq n$

(vi) there exists $\gamma_{0}: \Delta \rightarrow \mathbb{R}_{+}$such that

$$
\left|\varphi_{0}^{(i, m)}-\varphi_{0 \cdot h}^{(i, m)}\right| \leq \gamma_{0}(h) \quad \text { on } E_{0 \cdot h}, \quad \lim _{h \rightarrow 0} \gamma_{0}(h)=0,
$$

(vii) $\left.v\right|_{\Omega}$ is of class $C^{4}$,

(viii) there exists $\widetilde{d} \in \mathbb{R}_{+}$such that $\left\|\partial_{p} f(t, x, w, p)\right\| \leq \widetilde{d}$,

(ix) there exist $\widetilde{d}_{2}, \widetilde{d}_{4} \in \mathbb{R}_{+}$such that on $\Omega$,

$$
\left|\partial_{t t} v(t, x)\right|,\left|\partial_{x_{j} x_{k}} v(t, x)\right| \leq \widetilde{d}_{2}, \quad\left|\partial_{x_{i} x_{j} x_{k} x_{l}}^{(4)} v(t, x)\right| \leq \widetilde{d}_{4},
$$

Then there is $A \in \mathbb{R}_{+}$such that on $E_{h}$,

$$
\left|u_{h}^{(i, m)}-v_{h}^{(i, m)}\right| \leq \gamma_{0}(h) e^{\widetilde{L} a}+A\left\|h^{\prime}\right\|^{2} \frac{e^{\widetilde{L} a}-1}{\widetilde{L}}
$$

if $L>0$, and

$$
\left|u_{h}^{(i, m)}-v_{h}^{(i, m)}\right| \leq \gamma_{0}(h)+a A\left\|h^{\prime}\right\|^{2}
$$

if $L=0$, where $\widetilde{L}=L\left(1+n d_{2}\right)$.

Proof. We apply Theorem 1. Write

$$
\begin{aligned}
\psi_{h}^{(i, m)}= & \delta_{0} v_{h}^{(i, m)}-\sum_{k, j=1}^{n} \varrho_{k j}\left(t^{(i)}, x^{(m)}, T_{h} v_{h}\right) \delta_{k j}^{(2)} v_{h}^{(i, m)} \\
& -f\left(t^{(i)}, x^{(m)}, T_{h} v_{h}, \delta v_{h}^{(i, m)}\right) .
\end{aligned}
$$


As in the proof of Theorem 1 we can rewrite (46) as

$$
\begin{aligned}
\psi_{h}^{(i, m)}= & \delta_{0} v_{h}^{(i, m)}-\partial_{t} v\left(t^{(i)}, x^{(m)}\right) \\
& +\sum_{k, j=1}^{n}\left[\varrho_{k j}\left(t^{(i)}, x^{(m)}, v\right)-\varrho_{k j}\left(t^{(i)}, x^{(m)}, T_{h} v_{h}\right)\right] \delta_{k j}^{(2)} v_{h}^{(i, m)} \\
& +\sum_{k, j=1}^{n} \varrho_{k j}\left(t^{(i)}, x^{(m)}, v\right)\left[\partial_{x_{k} x_{j}}^{(2)} v^{(i, m)}-\delta_{k j}^{(2)} v_{h}^{(i, m)}\right] \\
& +f\left(t^{(i)}, x^{(m)}, v, \partial_{x} v\left(t^{(i)}, x^{(m)}\right)\right)-f\left(t^{(i)}, x^{(m)}, T_{h} v_{h}, \partial_{x} v\left(t^{(i)}, x^{(m)}\right)\right) \\
& +f\left(t^{(i)}, x^{(m)}, T_{h} v_{h}, \partial_{x} v\left(t^{(i)}, x^{(m)}\right)\right)-f\left(t^{(i)}, x^{(m)}, T_{h} v_{h}, \delta v_{h}^{(i, m)}\right) .
\end{aligned}
$$

There are $\widetilde{r}, d_{2} \in \mathbb{R}_{+}$such that

$$
\begin{aligned}
\left|\delta_{k j}^{(2)} v^{(i, m)}\right| \leq d_{2} & \text { on } E_{h}, 1 \leq k, j \leq n, \\
\left|\varrho_{k j}(t, x, v)\right| \leq \widetilde{r} & \text { on } E \times \mathbb{R}^{n}, 1 \leq k, j \leq n .
\end{aligned}
$$

From the Taylor formula we obtain

$$
\left|\partial_{t} v\left(t^{(i)}, x^{(m)}\right)-\delta_{0} v_{h}^{(i, m)}\right| \leq \frac{h_{0}}{2} \widetilde{d_{2}} \leq \frac{1}{2} \widetilde{d_{2}} \widetilde{c}\left\|h^{\prime}\right\|^{2}
$$

and

$$
\left|\partial_{x_{k} x_{j}} v\left(t^{(i)}, x^{(m)}\right)-\delta_{k j} v_{h}^{(i, m)}\right| \leq \widetilde{d}_{4}\left\|h^{\prime}\right\|^{2}\left(\frac{7}{12}+\frac{1}{6} c_{\star}\right) \quad \text { for } 1 \leq k, j \leq n .
$$

The above estimates and Lemma 2 yield

$$
\begin{aligned}
\left|\psi_{h}^{(i, m)}\right| \leq & \frac{1}{2} \widetilde{d}_{2} \widetilde{c}\left\|h^{\prime}\right\|^{2}+L \widetilde{d}_{2} d_{2} C_{0}\left\|h^{\prime}\right\|^{2}+\widetilde{r} \widetilde{d}_{4} n^{2}\left\|h^{\prime}\right\|^{2}\left(\frac{7}{12}+\frac{1}{6} c_{\star}\right) \\
& +L d_{2} C_{0}\left\|h^{\prime}\right\|^{2}+\frac{1}{6} \widetilde{d_{d_{2}}}\left\|h^{\prime}\right\|^{2} \quad \text { on } E_{h}^{\prime} .
\end{aligned}
$$

Thus on $E_{h}^{\prime}$,

$$
\left|\psi_{h}^{(i, m)}\right| \leq\left[\frac{1}{2} \widetilde{d}_{2} \widetilde{c}+L d_{2} C_{0}\left(1+\widetilde{d}_{2}\right)+\widetilde{r} \widetilde{d}_{4} n^{2}\left(\frac{7}{12}+\frac{1}{6} c_{\star}\right)+\frac{1}{6} \widetilde{d} \widetilde{d}_{2}\right]\left\|h^{\prime}\right\|^{2} .
$$

On $\partial E_{h}$ we have

$$
\left|v_{h}^{(i, m+\alpha)}-v_{h}^{(i, m-\alpha)}-2 \sum_{j=1}^{n} \alpha_{j} h_{j} \varphi_{j \cdot h}^{(i, m)}\right| \leq \widetilde{d_{3}}\left\|h^{\prime}\right\|^{3} .
$$

Thus all the assumptions of Theorem 1 are satisfied and assertions (44), (45) follow from (16), (17).

4. Numerical example. For $n=2$ we put

$$
E=[0,1] \times[-1,1] \times[-1,1],
$$


Let $z$ be the unknown function of the variables $(t, x, y)$ and consider the differential integral equation

$$
\begin{aligned}
\partial_{t} z(t, x, y) & =2\left(x^{2}+1\right) \partial_{x x} z(t, x, y)+2\left(y^{2}+1\right) \partial_{y y} z(t, x, y) \\
+ & \partial_{x y} z(t, x, y) \int_{(x-1) / 2}^{(x+1) / 2} \int_{(y-1) / 2}^{(y+1) / 2} z(t, \eta, \xi) d \xi d \eta \\
+ & 23 e^{t} x^{3} y^{3}-\frac{9}{64} e^{2 t} x^{2} y^{2}\left(x^{3}+x\right)\left(y^{3}+y\right)-12 e^{t} x y\left(x^{2}+y^{2}\right)
\end{aligned}
$$

with the initial boundary condition

$$
z(t, x, y)=x^{3} y^{3} \quad \text { for }(t, x, y) \in E_{0}
$$

and the Neumann boundary conditions

$$
\begin{array}{ll}
\partial_{x} z(t, x, y)=3 e^{t} x^{2} y^{3} & \text { for }(t, x, y) \in \partial_{0} E \text { and } x=1 \text { or } x=-1, \\
\partial_{y} z(t, x, y)=3 e^{t} x^{3} y^{2} & \text { for }(t, x, y) \in \partial_{0} E \text { and } y=1 \text { or } y=-1
\end{array}
$$

where

$$
\begin{aligned}
E_{0} & =\{0\} \times[-1,1] \times[-1,1], \\
\partial_{0} E & =(0,1] \times[([-1,1] \times[-1,1]) \backslash((-1,1) \times(-1,1))] .
\end{aligned}
$$

For the above problem we apply the difference method (37)-(39).

The function $v(t, x, y)=e^{t} x^{3} y^{3}$ is a solution of (47)-(50). Let $u_{h}$ : $E_{h}^{*} \rightarrow \mathbb{R}$ be the solution of the corresponding difference equations and $\varepsilon_{h}=u_{h}-v$. The values $\varepsilon_{h}\left(0.6, x^{(j)}, y^{(k)}\right), \varepsilon_{h}\left(0.7, x^{(j)}, y^{(k)}\right), \varepsilon_{h}\left(0.8, x^{(j)}, y^{(k)}\right)$, $\varepsilon_{h}\left(0.9, x^{(j)}, y^{(k)}\right)$ are listed in Table 1 for $h_{0}=0.00001, h_{1}=0.02$ and $h_{2}=0.02$.

Table 1

\begin{tabular}{rrrrrc}
\hline & & $t^{(i)}=0.6$ & $t^{(i)}=0.7$ & $t^{(i)}=0.8$ & $t^{(i)}=0.9$ \\
\hline$x^{(j)}$, & $y^{(k)}$ & $\varepsilon_{h}$ & $\varepsilon_{h}$ & $\varepsilon_{h}$ & $\varepsilon_{h}$ \\
\hline-0.5 & -0.5 & $6.090 \cdot 10^{-4}$ & $8.905 \cdot 10^{-4}$ & $1.271 \cdot 10^{-3}$ & $1.789 \cdot 10^{-3}$ \\
-0.5 & 0.0 & $5.040 \cdot 10^{-4}$ & $7.290 \cdot 10^{-4}$ & $1.033 \cdot 10^{-3}$ & $1.445 \cdot 10^{-3}$ \\
-0.5 & 0.5 & $5.558 \cdot 10^{-4}$ & $7.838 \cdot 10^{-4}$ & $1.094 \cdot 10^{-3}$ & $1.519 \cdot 10^{-3}$ \\
0.0 & -0.5 & $8.419 \cdot 10^{-4}$ & $1.149 \cdot 10^{-3}$ & $1.553 \cdot 10^{-3}$ & $2.091 \cdot 10^{-3}$ \\
0.0 & 0.0 & $5.684 \cdot 10^{-4}$ & $8.048 \cdot 10^{-4}$ & $1.120 \cdot 10^{-3}$ & $1.544 \cdot 10^{-3}$ \\
0.0 & 0.5 & $4.275 \cdot 10^{-4}$ & $6.337 \cdot 10^{-4}$ & $9.139 \cdot 10^{-4}$ & $1.297 \cdot 10^{-3}$ \\
0.5 & -0.5 & $1.425 \cdot 10^{-3}$ & $1.864 \cdot 10^{-3}$ & $2.436 \cdot 10^{-3}$ & $3.188 \cdot 10^{-3}$ \\
0.5 & 0.0 & $7.067 \cdot 10^{-4}$ & $9.815 \cdot 10^{-4}$ & $1.347 \cdot 10^{-3}$ & $1.835 \cdot 10^{-3}$ \\
0.5 & 0.5 & $3.678 \cdot 10^{-4}$ & $5.905 \cdot 10^{-4}$ & $8.982 \cdot 10^{-4}$ & $1.326 \cdot 10^{-3}$ \\
\hline & & & & & \\
\hline
\end{tabular}


Let $\varepsilon_{\max }$ be the largest and $\varepsilon_{\text {mean }}$ the mean value of all $\varepsilon_{h}$ for a given $t^{(i)}$. The values are listed in Table 2 .

Table 2

\begin{tabular}{ccc}
\hline$t^{(i)}$ & $\varepsilon_{\max }$ & $\varepsilon_{\operatorname{mean}}$ \\
\hline 0.0 & $1.157 \cdot 10^{-3}$ & $6.815 \cdot 10^{-5}$ \\
0.1 & $1.465 \cdot 10^{-3}$ & $1.127 \cdot 10^{-4}$ \\
0.2 & $1.957 \cdot 10^{-3}$ & $1.843 \cdot 10^{-4}$ \\
0.3 & $2.471 \cdot 10^{-3}$ & $2.705 \cdot 10^{-4}$ \\
0.4 & $3.075 \cdot 10^{-3}$ & $3.903 \cdot 10^{-4}$ \\
0.5 & $3.816 \cdot 10^{-3}$ & $5.549 \cdot 10^{-4}$ \\
0.6 & $4.738 \cdot 10^{-3}$ & $7.784 \cdot 10^{-4}$ \\
0.7 & $5.896 \cdot 10^{-3}$ & $1.080 \cdot 10^{-3}$ \\
0.8 & $7.364 \cdot 10^{-3}$ & $1.485 \cdot 10^{-3}$ \\
0.9 & $9.240 \cdot 10^{-3}$ & $2.032 \cdot 10^{-3}$ \\
1.0 & $1.166 \cdot 10^{-2}$ & $2.777 \cdot 10^{-3}$ \\
\hline
\end{tabular}

The computation was performed on a PC computer.

\section{References}

[1] Z. Kamont and M. Kwapisz, Difference methods for nonlinear parabolic differentialfunctional systems with initial boundary conditions of the Neumann type, Comment. Math. Prace Mat. 28 (1989), 223-248.

[2] Z. Kamont, M. Kwapisz and S. Zacharek, On difference-functional inequalities related to some classes of partial differential-functional equations, Math. Nachr. 146 (1990), $335-360$.

[3] Z. Kamont and S. Zacharek, Line method approximations to the initial-boundary value problem of Neumann type for parabolic differential-functional equations, Comment. Math. Prace Mat. 30 (1991), 317-330.

[4] M. Malec, Sur la méthode des différences finies pour une équation différentielle partielle non-linéaire parabolique sans dérivées mixtes avec la condition aux limites du type de Neumann, Bull. Acad. Polon. Sci. Sér. Sci. Math. Astronom. Phys. 22 (1974), 495-501.

[5] —, Sur une famille biparamétrique de schémas des différences finies pour un système d'équations paraboliques aux dérivées mixtes et avec des conditions aux limites du type de Neumann, Ann. Polon. Math. 32 (1976), 33-42.

[6] - Schéma des différences finies pour un système d'équations non linéaires partielles elliptiques aux dérivées mixtes et avec des conditions aux limites du type de Neumann, ibid. 34 (1977), 277-287.

[7] C. V. Pao, Finite difference reaction-diffusion systems with coupled boundary conditions and time delays, J. Math. Anal. Appl. 272 (2002), 407-434. 
[8] Z. Węglowski, On a difference method for non-linear parabolic equations with mixed derivatives, Zeszyty Nauk. Uniw. Jagiello. Prace Mat. 16 (1974), 161-167.

Institute of Mathematics

University of Gdańsk

Wita Stwosza 57

80-952 Gdańsk, Poland

E-mail: rciarski@math.univ.gda.pl

Reçu par la Rédaction le 10.2.2004 